\title{
A generic framework for simulation of supply networks with bargaining agents
}

\author{
N. B. Szirbik, G.R. Wagner and J.A. La Poutré, \\ Eindhoven University of Technology, Faculty Technology Management, Pav. D - 10, Postbus \\ 513, 5600 MB Eindhoven, the Netherlands. Email: N.B.Szirbik@tm.tue.nl
}

\begin{abstract}
In this paper we present the main aspects of the problem of simulation of a supply chain using an agent-based system. It is emphasized that the difference with other simulation models is that the principal concept of the simulation environment is the event concept. Agents are interacting via action/events, these concepts being introduced previously by the AOR modelling language. The configuration of the supply network is model based, the model being described in AORml. Because we use negotiation as a basis for agent-to-agent interaction, we specialise the AOR concepts in order to have a better mapping with the problems of supply network simulation. In a supply network, the negotiation is more bargaining (fallback) oriented, and we present some scenarios in this case, which clearly differentiate the generic model of interaction from the auction based one. We show how the experimentation with such a simulation test bed can be used to implement agent-based information systems for supply network management.
\end{abstract}

Keywords

Dynamic Agents, Simulation, Supply Chains.

\section{INTRRODUCTION}

In the last decade, many inter-disciplinary research projects have focused on the issue of applying agent technologies in the field of logistical coordination in various environments, as complex manufacturing enterprises, physical distribution of goods and supply networks. The interdisciplinary nature of this kind of research is given by the computer science and artificial intelligence background of the researchers who are proposing the agent technology and the management science and logistics background of the researchers in the area of application.

Using agent-based modelling, a supply network can be viewed as an environment where typically a limited number of moderately collaborative agents (representing enterprises) are operating. From the agent-oriented perspective, the main aspect of global operation of the network is interaction. Our choice for the main form of interaction is negotiation (auction mechanisms, fall-back bargaining, argumentation, etc). In a real-life setting of a supply network, humans carry out negotiation, or by IT support systems that always have a human-in-the-loop. 
On the other hand, completely automated negotiation is already a key form of interaction in some software multi-agent systems, like e-markets and active networks, where the number of agents is very high, the transaction should be performed as fast as possible and there is no form of collaboration between agents. Also, there is a very small chance that the same two agents will come often into contact. In contrast with e-markets, in typical supply chains, the community of agents is rather small, with partly common goals, while at the same time; there is competitiveness between the agents. Furthermore, there are repeated interactions between agents: the agents are not "anonymous". Therefore, particular solutions for negotiation are needed that take these different issues into account. An approach is the use of mediated interaction, in order to avoid loose-loose interactions between agents due to agent rationality (isolated "selfishness" of agents).

To study these issues, we propose an approach based on a simulation environment of a generic supply network, where the entities in the network are modelled and emulated as agents, and the interaction and logistic coordination are achieved by using software agents which play the role of the IT support system of the network.

The remainder of this paper is organized as follows. First, we motivate our approach and make a critical review of current supply network methods of research. Later we present the generic framework for modelling supply network. Then we present bargaining examples, in the light that this is our main meta-scenario for generic interaction. Finally we discuss the implementation and experimentation with the test bed.

\section{THE MOTIVATIONS: NON-REALISTIC MODELLING AND EXCESSIVE CENTRALIZATION}

A supply network (more commonly called "supply chain") is a network of legally independent enterprises and/or enterprise business units, which are playing the roles of suppliers, factories, warehouses, distribution centres, and retailers. These enterprises, depend on each other in the fulfilment of customer orders concerning products of a certain type. It is a great challenge today for computer science to develop the advanced information technology needed to support the coordinated planning and management of supply networks (more commonly called "supply chain planning" and "supply chain management").

Supply networks are playing an increasingly important role since modern manufacturing is moving away from vertically integrated companies that control all aspects of production and distribution, towards networks of autonomous enterprises participating in the order fulfilment process. The relationships between the nodes in a supply network are much looser than the relationships between different units of the same enterprise. They are based on social-economic mechanisms such as reputation; trust building, negotiation, contracting, social and economic rules of conduct, and national as well as international trade law. Although the business objectives of the involved parties may conflict with each other, all involved parties are committed to each other for jointly fulfilling customer orders. Completely selfish 
behaviour, like in competitive markets, is not possible since parties want to deal with each other in the future again.

For optimal performance, the nodes in a supply network must coordinate their demand forecasting, production planning and execution activities. But the dynamics of enterprises and markets make this difficult: materials do not arrive on time, production facilities fail, workers become ill, customers change or cancel orders, and so forth, causing deviations from the plan. Therefore, all plans and schedules must be revised periodically across the supply network in a coordinated manner. Centralized software solutions for supply chain planning are not able to deal with this dynamics created by unexpected events [Cohen and Lee, 1988], [Stadler et al, 2000]. Another obstacle that prevents central coordination in supply networks is that the management information system can only use partial information. Companies do not disclose their business information, which makes it difficult to maintain the central storage of a supply chain [Vermeer, 2001].

Decentralized, collaborative software solutions in the form of a multi-agent system, which are based on the concepts of autonomy and collaborative interactions, seem to be better suited to cope with the described problems. However, previous research in agent-based supply chain management has not really addressed the real problems. Agent-based systems for research purposes that have been developed so far are using a very simplified model of the supply chain, and moreover, in most of the cases, the supply chain itself is not modelled as an agent composed structure. One of the reasons for this is the complexity of the real-life examples of supply networks [Fox, Barbuceanu, and Teigen, 2001]. Also, many of the exceptions that occur in real-life environments, as order cancellations, delays, or accidents are not yet captured in the current models in the agent-based SCM research.

Our approach is to model first the supply network as a multi-agent system consisting of institutional, human, and software agents. This model has to capture the reality of the supply network as closely as possible, without abstracting away from essential aspects of the problem domain. Also, the model has to grow to a level where it can be executed, that is, gives the possibility to simulate the supply network, by running the software agents and simulating/emulating the rest of the physical agents.

In summary, we see as limitations of current approaches (1) the fact that the supply network is not modelled realistically, especially in terms of unexpected events, uncertain and incomplete information, complex interactions, etc, and (2) that the management model as well as the information model used in supply networks are too centralized.

\section{STATE OF THE ART IN SUPPLY CHAIN MODELLING AND AGENT NEGOTIATION AND MEDIATION}

In previous research in the domain of supply network management, three different approaches have been used for the problem of modelling and analyzing the supply chains:

1. Control theorists model the chain with differential or difference equations and use transform analysis to explore their behaviour. This approach relies heavily 
on linearity assumptions that are not satisfied in most supply networks (as described in [Towill, 1991]).

2. Classical operations research approaches include optimization theory, game theory, and statistical analysis. These tools apply to nonlinear as well as linear systems, but often make unrealistic assumptions. In addition, they are not explicitly modelling the iterative nature of supply chain planning, and so cannot capture the dynamical characteristics of the system (see [Lee, Padmanabhan and Whang, 1997]). This line of research typically assumes a kind of centralized control of the supply network, and does not explore the possibilities of decentralized decision-making. The new "classical" chain models in this area include [Cohen and Lee, 1988], [Cohen and Moon 1990], [Lee and Billington, 1993], and [Pyke and Cohen, 1994]. However, these models are limited in handling issues related to the dynamics of supply networks and focus exclusively on global performance measures.

3. Simulation approaches experiment with an executable model of the system. In most cases these models are in support of one or the other of the previous two approaches. Most of the simulation work models the supply chain as a static set of partial differential equations and then integrates these equations over time (for a literature review of all of these three approaches, see [vd Parunak et al.1998]).

The use of simulation as a means for evaluating dynamic supply chain management has gained considerable attention and momentum in recent years (for an overview, see [Feigin et al. 1997]). In [Tzafestas and Kapiotis, 1994], a method combining an analytical/simulation model was used to analyze the problem of demand amplification in complex supply network settings. Agent-based simulation was first used by [Swaminathan, Smith and Sadeh, 1995] to study the effect of sharing suppliers' available-to-promise (ATP) information.

Our research focuses on two issues that have only been peripherally investigated in other agent-based approaches, namely negotiation and mediation. Using negotiation as a coordination technique in distributed systems dates back to the Contract Net Protocol proposal of [Smith, 80], [Davis and Smith, 83] and [Smith and Davis, 1988]. This is basically a reverse auction based scheme. One of the first agent-oriented approaches that studied auction based negotiation in distributed planning was the seminal work of [Moehlman, Lesser, and Buteau, 1992]. Today, the same laboratory (Fundamental and Applied Research in Multi-agent Systems $\mathrm{Lab}$, at University of Massachusetts at Amherst), pursues projects for "Modelling Coopetition (Cooperative Competition) in Distributed Supply Networks" [FAR], where the auction mechanisms are viewed as the most important issues. However, negotiation in their context is represented still by the same four auction schemes used in the past: the English auction, the Dutch auction, the sealed auction and the Vickrey auction (see [Krothapalli \& Deshmukh, 1999]). Multi-item auctions (socalled combinatorial auctions) for planning have been studied in the same group, [Sandholm \& Lesser, 1995] but the appearance of the new field of e-markets has driven many researchers studying the auction-based negotiation from the supply chain problems to the new field. Good examples for this new trend are the AIRV laboratory at University of Minnesota at Minneapolis (the group for Intelligent 
Agents for e-Commerce, developing the MAGNET research test bed, [MAG]) and the LAIT laboratory at University of Maryland Baltimore County [LAI]. In [Collins et al., 2000], auctioning agents configure a supply network and its plans, but these agents are "selfish", purely rational agents, which try to maximize their profit, and no collaborative behaviour is added. [Peng et al., 1999] presents a more refined model of negotiation, but this approach is rather focused on the design of the negotiating protocols than the study of the overall dynamics of a supply network controlled by these agents. Another relevant contribution in the field of negotiation is the work within the group of Jennings at Southampton University (see [Jennings et al 2001]. In [Fatima, Wooldridge \& Jennings 2001]) negotiation is made with incomplete information and also is studied how time can affect negotiation. In our view, the most important development is that the auctioning party (who is basically a mediator) can help to compute the winner in multi-item auctions.

The concept of mediation was first used in [Maturana \& Norrie 1995]. A mediated agent-based model for supply networks is proposed in [Swaminathan 1996]. Their model is used to address issues related to coordination and contracts. The Centre for Research in Electronic Commerce, Austin, has examined also a supply chain information system as a collection of agents [Hinkanen et al. 1997], where some agents responsible for the products in the supply network and the rest are a sort of mediators (electronic brokers). Another mediated approach for the negotiation between agents in a supply network model is used also at the Enterprise Integration Laboratory in Canada [Fox, Barbuceanu \& Teigen 2000]. They call the mediators logistic agents, these being responsible for the overall coordination. The Intelligent Coordination and Logistics of CMU, Pittsburgh, proposed an architecture called MASCOT [Sadeh 1996], which was extended with negotiating and mediating techniques in [Kjenstadt 1998]. There, a new formal concept of "fairness" is developed (continued in [Szirbik 2002] with the issue of mediator "neutrality").

Mediated peer-to-peer negotiation was studied in [Tesch \& Fankhauser, 1999], but only for market environments, where interactions between the same pair of members are seldom. Also, the method of research was based on game theory and it is hard to be applied for distributed planning systems. For supply networks (of a opportunistic kind), a mediated scheme of negotiation between small groups of agents was first proposed in [Szirbik et al. 2000], and it is based on the "fall-back" bargaining scheme. Fall-back bargaining was studied previously (for an overview, see [Raiffa, 82], and for a multi-criteria negotiation see an overview in [Brams \& Kilgour, 2001]), but the implications of using mediators in this mechanism applied in a supply network community of agents have been thoroughly addressed for the first time in [Szirbik 2002].

The software industry also has an emerging, substantial interest in agentoriented approaches for supply chains. Examples are Invesys Baan ltd. and SAP AG who are offering today "agent-oriented" software components for supply chain management. Even these are using only the most primitive aspects of the agent technology, as reactivness and some pro-activeness, together with some learning/adaptive methods; it is a very important development, showing that slowly the industry is accepting this new paradigm.

A powerful modelling framework can enhance the way the supply network is represented for simulation. We also consider that another step forward to achieve 
realistic modelling is the usage of fallback bargaining, because in the reality, auctions are seldom used. However, to have an agent-based system accepted in a real-life environment, the enterprises that will use the system will desire a neutral and fair behaviour of the system. To explicitly include this in the generic model of the agent-based system, we consider that a neutral mediation agency should exist "de rule".

\section{THE MODELLING FRAMEWORK}

We consider that the modelling of both the experimentation system (test bed) and the supply chain network is equally important as the implementation of the system and the experimentation phase. First, the problem of modelling the supply network as a multi-agent system is addressed by selecting the appropriate physical and information entities that will be modelled as agents. At a generic level, we use a three-type agent model, consisting of human, institutional and information (software) agents.

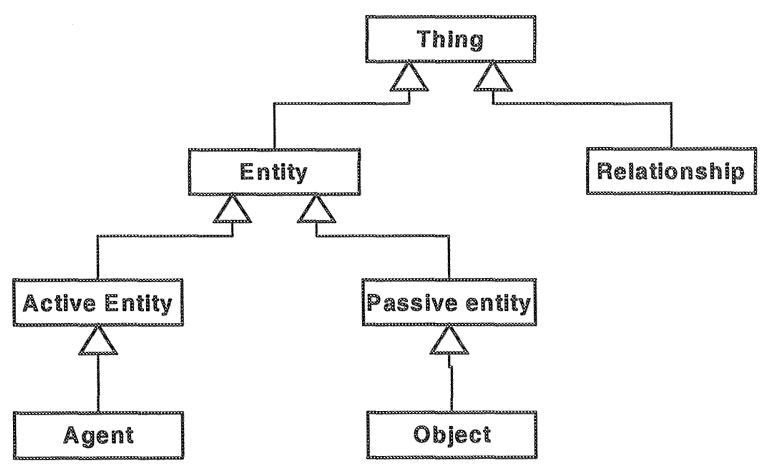

Figure 1 Basic AOR Concepts

In order to provide a strong formal basis for the modelling a supply network with agents, we have extended the AOR meta-model [Wagner 2002]. In this metamodel, the world is seen as a collection of agents, objects and relations. From an ontological perspective, starting with the classical E-R (entity-relationship) ontology, the taxonomy of the basic AOR concepts is given in Figure 1. The concepts of Active and Passive Entity can be taken out from this classification; their role here is just to show the ontological principle that is differentiating between mere objects and agents.

The three concepts which are the leafs in this classification, are the three metaclasses which are used in developing AOR models. These models have an UML basis, with the difference that entities which show special behaviour (as proactiveness and "social" skills, like pattern and language based interaction) are modelled in a different way as mere objects, which are purely reactive and the communication with them is based only on interface-based invocations. In our definition for agents, we consider also that pro-active and protocol based software 
entities (components) can be included in the agent definition. Because in the UML diagrams there are no distinctive descriptions for agents, in AOR, the agents are depicted as rectangles with rounded corners. For example, an AOR agent-class diagram depicting the structure of a simple supply network is given in Figure 2.

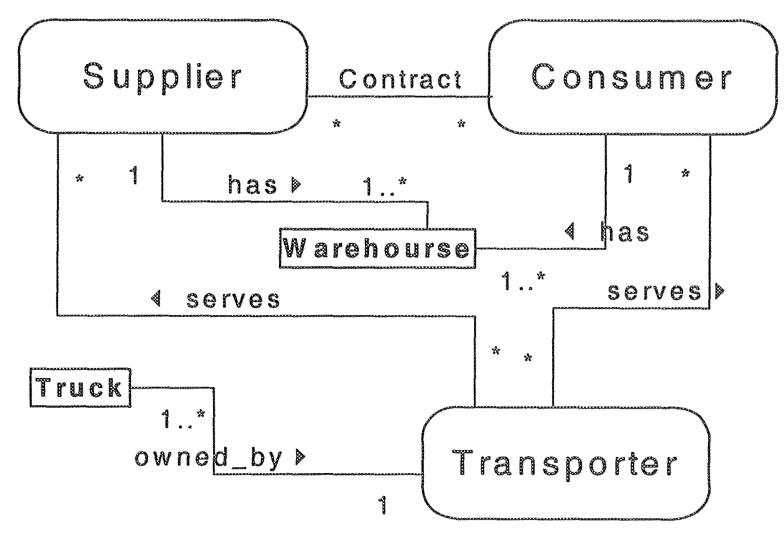

Figure 2 - An Example of Simple Supply Networks

The problem is that a supply network is a complex system, where enterprises, humans and various software components are interacting and acting on various physical and abstract objects as products, contracts, orders, commitments, etc. To capture in a better way the reality, we use a refined form of the AOR taxonomy with three new types of agents, as in Figure 3. In the original AOR metamodel, the agent concept is specialized in three types of agents: biological agents (usually humans), artificial agents (software components and robots), and institutional agents, which can model organizations (enterprises or enterprise units). In this paper, a supplementary concept is added in the taxonomy, that of an atomic agent. Also, we consider that in the supply network universe of discourse, we have only human biological agents and only software artificial agents (we are not considering physical artificial agents, as autonomous robots to be yet part of the models).

In order to separate the graphical description of the three basic agent types, we use the stereotypes depicted in Figure 3. The taxonomy presented in Figure 4 implies a strong ontological commitment, that is, a software agent is always considered an atomic agent, and in other words, we cannot have a software agent that is composed by other software agents. Perhaps this choice seems to be very rigid, but the possibility to compose complex artificial agents is given by the existence of institutional agents.

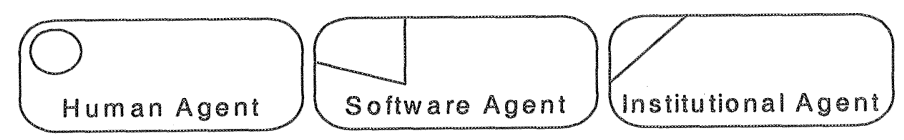

Figure 3 Three Basic Types of Agents 


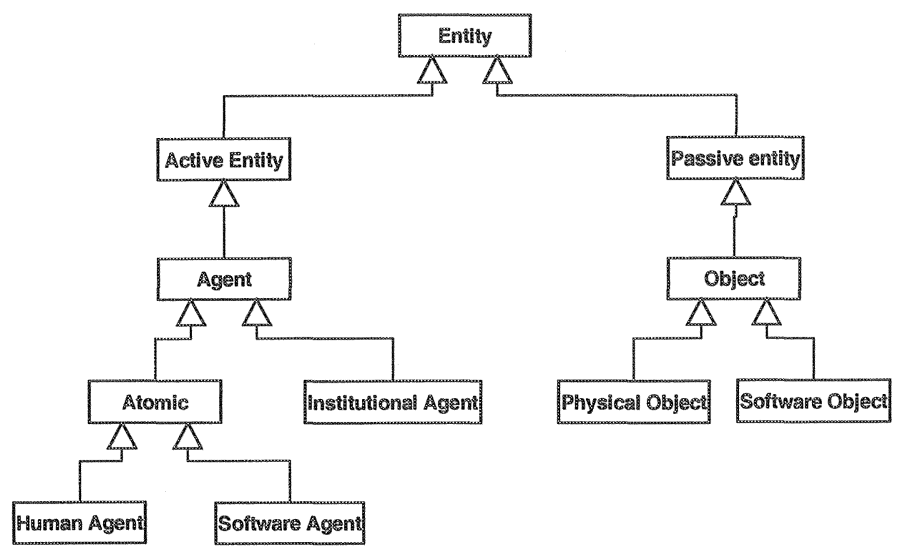

Figure 4 Taxonomy of three Basic Types of Agents

Completing the AOR meta-model, some new principles are introduced:

1. A software agent has always to be owned always by an institutional agent

2. An institutional agent is composed at least by one atomic agent and has a predefined meta-model (see Figure 5)

3. Any modelled system can be viewed as an institutional agent, that is, any objects may be owned by or linked to another entity.

Because the institutional agent is a non-atomic agent, its designated (predefined) structure should be described. There are two ways to describe this: the external perspective [Wagner 2002], used in the domain models, and the internal perspective, which is used for the implementation design. The institutional agent's pre-defined meta-model, is shown in both these perspectives in Figure $5(\mathrm{a}, \mathrm{b})$.

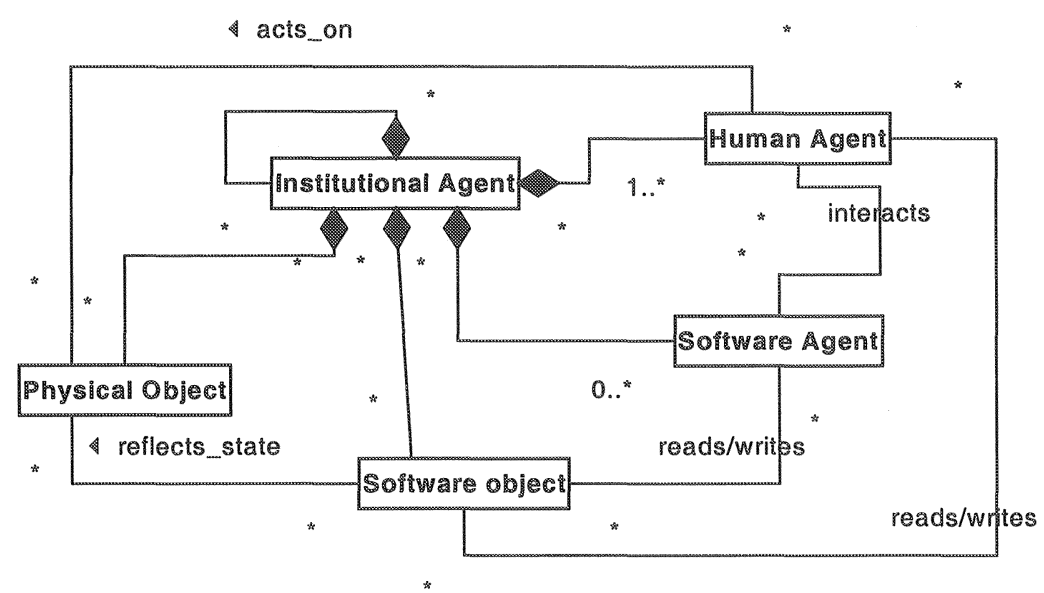

Figure 5 a The external perspective (model used for the implementation design, here in UML) 


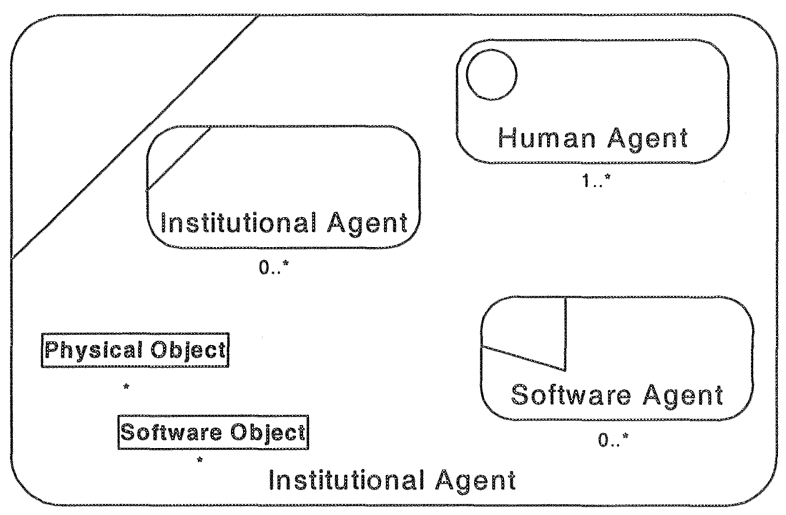

Figure $5 b$ The internal perspective (used to describe the domain model)

As presented here, an institutional agent must have at least one internal human agent, and can have zero or more software agents. This prohibits the existence of purely artificial institutional agents, but we will not consider yet such types of organization in our supply network modelling.

The use of the AOR metamodel solves the problems of how interaction and communication is modelled (for an exhaustive explanation, see [Wagner 2002]). Entities as action/event and claim/commitment and designated relationships as perceive, send, receive, does are used in special diagrams (interaction frame diagrams and interaction sequence diagrams) that capture the dynamics of agent interaction and communication. However, AOR is intended to be used more in information systems engineering than in software engineering processes. A natural approach would be to model the supply network using AOR in the first phase and to develop an object oriented model of the adjacent information system in the second phase, starting with the internal model described in AOR. Unfortunately, a purely OO model of the information system will not make any distinction between the software objects and the physical objects and more than that, it will not allow a special description for the software agents. The use of software agents in a supply network is the main focus of our research and the main reason to build the test bed. Because of this, we need in this particular research a supplementary set of ontological principles, which apply only in the case of the simulative experimentation.

In the executable model of the supply network (that is, the simulated supply chain) that will be developed, we will have two kinds of agents. The first kind simulates the physical agents in the supply network (enterprises/units and humans), and the second kind emulates the agents in the information system for supply network. Because the whole system can be considered as an institutional agent, we can include the users themselves in this agent, as internal human agents. A new taxonomy of the entities implied by this simulation is depicted in Figure 6. There are two basic kinds of entities involved: real and virtual. In our case, the real entities are the human experimenters. The virtual entities are all the rest of the simulated and emulated objects and agents. 


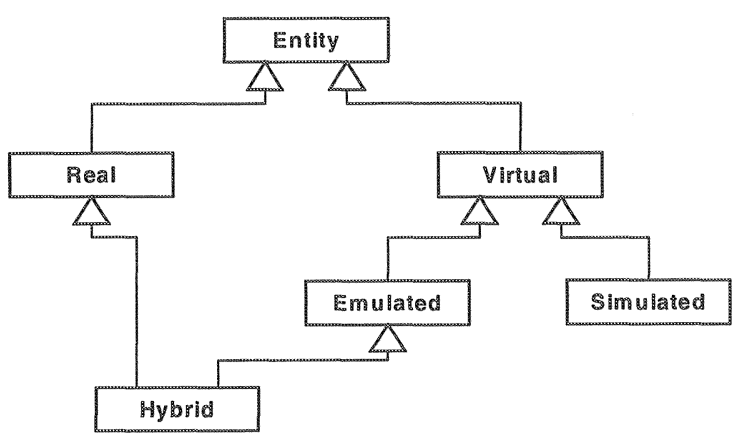

Figure 6 - A new taxonomy of the entities implied by simulation

If this taxonomy is combined with the previously discussed concepts, the software components that are part of the test bed will have the following relation with the taxonomy in Figure 3:

- the supply network will be an institutional agent which is simulated by the test bed (SNAg - the supply network agent)

w the enterprise/unit, which is a node in the supply network, will be a simulated institutional agent, internal to SNAg

- the humans in the enterprises, will be hybrid agents in the test bed. That is, some of their behaviour will be implemented in software, but the experimenters can also intervene and play their role, when the complexity of the decision making is leading to computationally impossible implementations

- the test bed software agents will emulate the software agents. In principle, their functionality can be upgraded to real-life setting levels, and in this case, these can be eventually deployed in a supply network

w the software objects (databases, e-documents, workflow systems, etc), will be emulated objects

a the physical objects will exist in the test bed as simulated objects

The reason why the human agents are hybrids is because it is extremely difficult to simulate realistic behaviour of human agents. The simplest way to overcome this is to use real human behaviour in some certain exception situation.

Also, we identify two kinds of real entities: the user-type experimenter and the designer-type experimenter. In an experiment, more than one of these experimenters can have a seat. We mentioned that this kind of research is multidisciplinary, having a twofold approach: from computer science and artificial intelligence and from logistics and operations research. The user-type experimenter will be the logistical expert, who will try to run various logistic scenarios, verify various logistical coordination mechanisms and study in general the overall behaviour of the supply network from the operational and performance points of view.

The designer-type experimenter will have the role to verify and adapt the behaviour of the emulated software agents, and eventually the behaviour of the simulated human agents. Also, he has to identify with the help of the user-type experimenter the necessary rules of the simulated institutional agents. The development of the system will go through the following phases: implementation 
and pre-experimentation. The implementation will bring a system that can simulate a variety of supply networks and agent behaviours. The simulation models should be built via automatic code generators and graphical interfaces with easy-to-use graphical languages.

The pre-experiment phase of the test bed will consist of the following steps:

1. modelling a generic supply network (the user-type experimenter can change the structure of the network)

2. modelling the physical objects in the supply network

3. modelling the software objects in the supply network

4. modelling the behaviour of the institutional agents

5. modelling the partial behaviour of the human agents

6. modelling the basic behaviour of the software agents

7. checking the coherence of the resulted executable model

It is important to note that the test bed should be easily re-configured with a new business model in step a. We will give an example of such a model later in this paper. An experiment using the test bed will consist off:

1. establishing an experiment scenario, inspired from real life settings

2. setting the structure of the network

3. an eventual upgrade (or selection) of the behaviour of the software agents

4. setting the initial state (stocks, lead-times, capacities, etc.)

5. starting the simulation

6. produce unexpected events

7. supply human behaviour if necessary

8. monitor the evolution of the system

In the simulation-based research, problems from real cases in supply networks will be used for experiments. Above the scenario, we can define special kinds of interaction between the business units in the supply network. Because of our previous interest in fallback bargaining and third trusted party mediators, we intend to conFigure our test-bed in the pre-experiment phase in a way that will allow us to find the best mechanisms for bargaining and the best techniques that insure neutral mediation.

\section{BARGAINING AND MEDIATION AS META-SCENARIOS}

It is worth to examine the fallback bargaining protocol-based interaction [Szirbik, 2002] in an agent-based supply network because of the following reasons:

- This protocols mimics better than the auction protocol the real inter-enterprise business processes in a supply network

- It is more probable to achieve win-win outcomes than other business interaction protocols [Fankhauser and Tesch, 1999]

* The protocol can be easily automated by using software agents, which can be provided by a third trusted party 
This third trusted party can also offer advice in selecting the appropriate bargaining strategy, and also can offer neutral mediation service. In an opposite approach, using auctioning mechanisms, the agents are merely competing with each other and there is no room for enacting a central service provider, excepting for the auctioneer, which is a rather passive role, eventually detecting bidders' collusion tactics.

To illustrate the usefulness of the previously presented modelling method, and also to show how bargaining processes become building blocks in a supply network scenario, we present here an example of an event-handling in a three node supply network. There are three institutional agents involved: a supplier $\mathrm{A}$, a consumer $\mathrm{B}$, and a transporter $\mathrm{C}$. A product $\mathrm{X}$ has to be transported from $\mathrm{A}$ to $\mathrm{B}$ by $\mathrm{C}$ in the time interval [d1..d2], where $\mathrm{d} 1$ is the date/time to pick up $X$ from $A$ and $d 2$ is the date/time to deliver $X$ to $B$. Suppose that $C$ has a problem and has to postpone/prolong the pick-up and delivery of $\mathrm{X}$, and the new time interval is $[\mathrm{d} 1+\mathrm{dp} . \mathrm{d} 2+\mathrm{dd}]$, where $\mathrm{dp}$ is the delay in the pick-up, and dd the delay for delivery. There can be a context in which $B$ will suffer because of the delay, and the transporter should pay the cost of the delay. In another context, there are no delay effects, but still B will want to have delay fees paid by C. The agents that will negotiate, should detect the context and use appropriate bargaining strategies. In a special context, $\mathrm{B}$ can even reward the delay, if the internal process in B using $\mathrm{X}$ has to be delayed due to some local events.

The same line of reasoning can be applied to the negotiation between $\mathrm{A}$ and $\mathrm{C}$. We assume in this example that the realization of $\mathrm{X}$ is desired by $\mathrm{A}$ to be postponed (due to other high-priced rush orders that are competing with the resources used for $\mathrm{X}$ ) and $\mathrm{A}$ is willing to pay $\mathrm{C}$ a reward for the delay in picking-up $\mathrm{X}$. To model this situation with an agent diagram (Figure 7), we have first to identify the AOR agents that are present in this situation. There are three institutional agent $\mathrm{A}, \mathrm{B}$, and $\mathrm{C}$, and we consider that we have three human agents within each of the institutional agents (a salesperson in A, a purchasing manager in B and a fleet manager/planner in C). Each institutional agent will be provided with a set of software agents, mainly for the negotiation process, but also for planning. These planning agents will have the capability to assess the damage created by a disturbance in the plans.

The scenario can unfold in the following way:

* The simulated agent $\mathrm{fm}$ (fleet manager) perceives that the transportation process of $\mathrm{X}$, from $\mathrm{A}$ to $\mathrm{B}$ is delayed by an external event (produced by the user-type experimenter).

- A supplementary step is to announce the delay to A and B (either fm is doing this directly by phone, fax email to $\mathrm{sp}$ (salesperson) in $\mathrm{A}$, and pp (purchasing person) in B, either delegates a software agent (not shown in Figure ?) to announce other corresponding software agents in $A$ and $B$.

- Agent $\mathrm{fm}$ delegates the (emulated) software agent ag_C1 to negotiate a delay fee with the corresponding agent at $\mathrm{B}$ (ag_B 1 ) and eventually initiate a reward negotiation process with the corresponding agent $\mathrm{C}$.

- Agent ag_C1 starts fallback bargaining with ag_A1 and ag_B1.

w Agent ag_B1 asks agent ag_B2 the cost of the delay. The same can happen in $\mathrm{A}$, but for example, agent ag_A1 can ask human agent sp about a possible amount for the reward. 
When in both threads of negotiation an agreement is reached, the process stops.

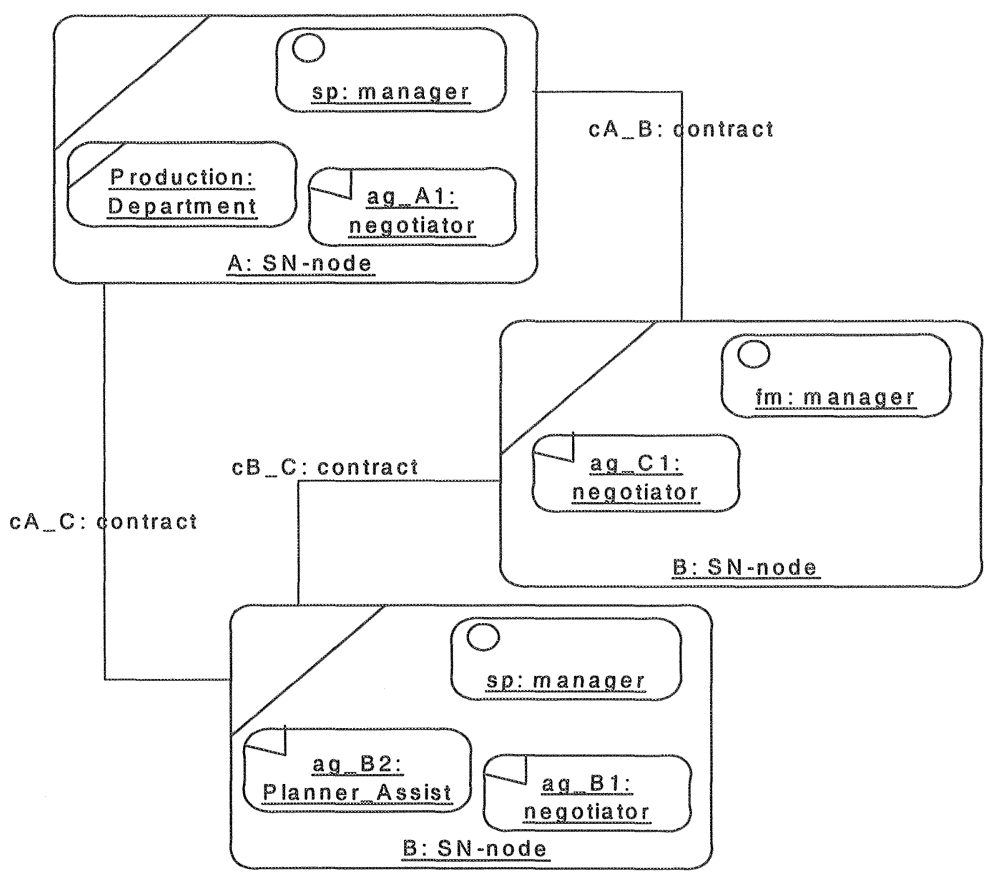

Figure 7 Institutional Agents

There are some observations related to this scenario:

- Agent ag_C1 should be able to perform and combine (balance) dynamically the negotiation processes. On the other hand, this is not a hard constraint, because the negotiations can take place sequentially - first negotiate the loss, and then try to balance it with the reward, for example.

- The contracts between the parties should not specify fixed fees for delays. This is based on the observation that the cost of the delay is very much context dependent.

- The delay problems should be solved as much as possible by the emulated software agents ag_A1, ag_B1, and ag_C1. However, when the problem becomes too complex for their logic, or the decision to be taken is considered too important or too risky to be delegated, the human agents can take charge. "His" decision is based either on the logic coded in the simulated human agent, or it can be provided by the experimenter.

Our insight is that during repeated experimentation with various scenarios, many of the external human experimenter interventions (which will be logged), can be transformed into empirical decision-making patterns, which can be coded as decision-making mechanisms into the simulated human agents. Of course, the human experimenter can monitor step by step the simulated human agents behaviour and overrule decisions when $\mathrm{s}(\mathrm{he})$ considers this appropriate. 
From the computer science perspective, the main role of the test bed is to discover what kind of "functionality" and decision-making can be transferred/delegated from the human agents to the software agents. The software agents can execute Tedious, but simple and repetitive/routine tasks, activities that need ultra real-time resolution. An approach is to develop a first version of the test bed with very simple software agents (based on finite state automata - FSA), and with basic rule-based simulated human agents, which contain only few rules, mainly to detect exceptions and invoke the help of the experimenters. Later, the complexity of the agent implementation can be increased, by using methods presented in the next section.

\section{IMPLEMENTING AND TESTING EASY CHANGEABLE AGENT BEHAVIOUR}

We envisage that the first hurdle of this research will be to insure a coherent behaviour of the overall simulation of the multi-agent system. Some steps in this direction have been made in [Szirbik and Wagner, 2001.]. An elegant way to implement adaptive behaviour of agents is by finite state automata (FSA). In the project, several approaches will be investigated, like e.g. hybridization with neural networks (NNs) for non-deterministic, adaptive behaviour (where the adaptive weights in the NN are attached to the FSA transitions), or like reconfiguration of the transition structure of the FSA using genetic algorithms (GAs).

An advanced, natural way to model adaptive behaviour that will be investigated is based on rules. Inference based on rules can be extended with neural weights representing uncertainty factors, and fuzzy linguistic variables, whose distribution functions are continuously adjusted using $\mathrm{NN}$ or GA algorithms. Then, a mapping will be established between the targeted concepts that are to be represented/handled as soft computing variables. This is especially feasible since the real concepts from supply chain also have a similar soft nature, such as for fair prices, loose delivery dates, flexible inventory levels, scrap factors, but also more complex concepts as trust values and mediator neutrality measure.

Rule-based agent systems can be difficult to be tested and validated. Workflows (at a formal level: Petri nets) can model parallelism within one agent execution. In hierarchical multi-agent systems, these models allow formal and semi-formal verification of local and global behaviours. For rule-based agent systems, one of the methods is to translate the rules in Petri nets (in [Szirbik and Wagner 2001] the "commitment-claim" agent related concept was applied to facilitate this translation). Because, in addition, the above soft-computing methods induce a non-deterministic behaviour, it is necessary to establish new methods to verify and test the soft rule based agent model. We consider deriving such methods from Petri net-based model checking and statistical analysis. 


\section{CONCLUSIONS}

We believe that the best method to study and develop the behaviour of software agents in any application is to simulate the entire environment as a single institutional agent, composed by emulated software agents and software objects, simulated institutional agents, and hybrid (simulated and impersonated) human agents. The experimentation can start with an empty shell, where the behaviour of the simulated humans will be enriched with various rules, heuristics and behavioural patterns. Step by step, the routine and simple to model actions can be transferred to the software agents, which became empowered to take decisions taken previously by the humans. In order to prepare the simulative experimentation in an easy way, we needed a generic framework, used for modelling the supply network as a collection of agents of various types. The role of the simulation is twofold, to study various supply network phenomena, in the light of event driven simulation, and to identify and develop the complex behaviour of the agents that can play finally the role of the supporting ICT system for a real supply network.

\section{REFERENCES}

[1] Brams, Steven J. and Kilgour, Marc D. (2001), Fallback bargaining, Group Decision and Negotiation 10, p287 - 317.

[2] Cohen, Morris, A. and Lee, Hau, L. (1988), Strategic analysis of integrated productiondistribution systems: Models and methods, Operations Research, 36(2), p216- 228.

[3] Cohen, Morris, A. and Moon, S. (1990), Impact of Production Scale Economies, Manufacturing Complexity and Transportation Costs on Supply Chain Facility Networks, Journal of Manufacturing and Operations Management, 3, p269 - 293.

[4] Collins J., R. Sundareswara, M. Tsvetovat, M. Gini, and B. Mobasher, (2000), "MultiAgent Contracting for Supply-Chain Management", Technical Report 00 - 010 , University of Minnesota. Available (in pdf) from http: //www.cs.umn.edu/tech_reports/.

[5] Davis, Randall and Smith, R.G. (1983), Negotiation as a metaphor for Distributed Problem Solving, Artificial Intelligence, 20: 69 - 109.

[6] Euwe, Mark (1999), Decentralized Control Systems for Logistical Coordination, Phd Thesis, BETA Research Institute for Operations Management and Logistics, Eindhoven University of Technology.

[7] Tesch, T. and Fankhauser, P. (1999), Arbitration and matchmaking for agents with conflicting interests, in Proc of Intl Workshop on Cooperative Information Agents (CIA99), Uppsala, Sweden, p323 - 334.

[8] [FAR] http: //farm.ecs.umass.edu/projects.html

[9] Fatima, S. S., M. Wooldridge and N. R. Jennings (2001) "Optimal negotiation strategies for agents with incomplete information" Proc. 8th Int. Workshop on Agent Theories, Architectures and Languages (ATAL), Seattle, USA, 53 - 68.

[10] Feigin, G.C. Connors, D. and Crawford, I. (1996), Shape Up, Ship Out, OR/MS Today, 4, p24-30.

[11] Fox, Mark, S. Barbuceanu, M. Teigen, R. (2000), Agent-Oriented Supply-Chain Management, Intl. Journal of Flexible Manufacturing Systems, 12, p165 - 188.

[12] Hinkkanen, A., R. Kalakota, P. Saengcharoenrat, J. Stallaert and A.B. Whinston, "Distributed Decision Support Systems for Real-Time Supply Chain Management," Chapter 12 in Readings in Electronic Commerce (ed. R. Kalakota and A.B. Whinston), Addison Wesley 1997, pp. 275 - 291. 
[13] Jennings, N. R., P. Faratin, A. R. Lomuscio, S. Parsons, C. Sierra and M. Wooldridge (2001) "Automated negotiation: prospects, methods and challenges", Int. J. of Group Decision and Negotiation, 10 (2) $199-215$.

[14] Kjenstadt, Dag (1998), PhD Thesis, Norvegian University of Science and Technology, Department of Production and Quality Engineering, Trondheim, and Carnegie Mellon University, Pittsburgh (NTNU rapport 24: 1998).

[15] Krothapalli, N. and Deshmukh, A. (1999), Strategic Coalition Formation in Supply Chains, see at [FAR] (http: //farm.ecs.umass.edu/publications).

[16] [LAI] http: //www.cs.umbc.edu/lait/

[17] Lee, Hau, L. Padmanabhan, V. and Whang, S. (1997), Information distortion in a Supply Chain: The Bullwhip Effect, Management Science, 43(4), p546 - 558.

[18] Lee, Hau, L. and Billington, C. (1992), Material management in decentralized supply chains, Operations Research, 41(5), p835 - 847.

[19] [MAG] http: //www-users.cs.umn.edu/ gini/magnet.html

[20] Maturana, Francisco, P. and Norrie, D.H. (1995), "A Generic Mediator for Multi-Agent Coordination in a Distributed Manufacturing System, Technical Raport, University of Calgary (at http: //ksi.cpsc.ucalgary.ca/projects/Mediator/)

[21] Moehlman, T., Lesser, V. and Buteau, B., "Decentralized Negotiation: An Approach to the Distributed Planning Problem," Group Decision and Negotiation 1: 2, K. Sycara (ed.), pp. 161 - 192. Norwell, MA: Kluwer Academic Publishers, 1992.

[22] Parunak, vd H. Savit R. Riolo R.L. "Agent-Based Modelling vs. Equation-Based Modelling: A case study", in Proc of MABS'98, Springer (http: //www.erim.org/ van/mabs98.pdf).

[23] Peng, Y. Finin, T., Labrou, Y. and Cost, R.S. (1999), An agent-based approach for manufacturing integration - the CIMPLEX experience, Applied Artificial Intelligence, 13: 39 - 63.

[24] Pyke, David F. and Cohen, Morris, A. (1993), Performance characteristics of stochastic integrated production-distribution systems, European Journal of Operations Research, $68(1), \mathrm{p} 23-48$.

[25] Raiffa, H., (1982), The Art and Science of Negotiation, The Belknap Press of Harvard University Press, Cambridge, MA.

[26] Sadeh, Norman M. (1966), MASCOT: An Agent Architecture for Multi-level Mixed Initiative Supply Chain Coordination, Technical Raport, Intelligent Coordination and Logistics Laboratory, Carnegie Mellon University.

[27] Sandholm, T. and Lesser, V. 1995. Issues in Automated Negotiation and Electronic Commerce: Extending the Contract Net Framework. First International Conference on Multi-agent Systems (ICMAS-95), San Fransisco, pp. 328 - 335 (ftp: //ftp.cs.umass.edu/pub/lesser/sandholm-icmas95-issues.ps).

[28] Smith, Reid, G. (1980), The Contract Net Protocol: High-level communication and control in the distributed problem solver, IEEE Transaction on Computers, 29(12): 1104 $-1113$.

[29] Smith, Reid, G. and Davis, R. (1988), Framework for Cooperation in Distributed Problem Solving, in Readings in Artificial Intelligence (eds. Bond and Gasser), Morgan Kaufman, CA, p61 - 70.

[30] Stadtler, H. and Kilger, C., 2000, Supply Chain Management and Advanced Planning : Concepts, Models, Software and Case Studies, Springer, Berlin.

[31] Swaminathan, Jayashankar M. (1996), Quantitative analysis of emerging practices in supply chains, PhD Thesis, Graduate School of Industrial Administration, Carnegie Mellon University.

[32] Swaminathan, J.M., S.F. Smith and N.M. Sadeh (1998), "Modelling Supply Chain Dynamices: A Multi-Agent Approach", Decision Sciences, 29(3).

[33] Szirbik, N., Wortmann, H., Hammer D., Goossenaerts, J.B.M., Aerts, A.T.M. (2000), "Mediating Negotiations in a Virtual Enterprise via Mobile Agents", Proc. of Academia- 
Industry Working Conference on Research Challenges (AIWORC 2000): "Next Generation Enterprises: Virtual Organizations and Mobile / Pervasive Technologies", (eds. Rao and Ramesh), SUNY at Buffalo, NY, USA, April 2000, IEEE Computer Society Press.

[34] Szirbik, N.; Wagner, G.: Steps Towards Formal Verification of Agent-based E-Business Applications. In Moldt, D. (ed.): Proceedings of Workshop on Modelling of Objects, Components, and Agents (MOCA'01),27-28 August 2001, Aarhus, Denmark. Datalogisk Institut Denmark, Aarhus, 2001, pp. 133-142.

[35] Szirbik, N., (2002) "A Negotiation Enabling Agent Based Infrastructure: Composition and Behaviour", in Information Systems Frontiers, (Kluwer Academic Journals),

[36] Towill, D.R. (1991), Supply Chain Dynamics, Intl. Journal of Computer Integrated Manufacturing, 4(4), p197 - 208.

[37] Tsafestas, S. and Kapsiotis, G. (1994), Coordinated control of manufacturing/supply chains using multi-levels techniques, Computer Integrated Manufacturing Systems, 7(3), p206-212.

[38] Vermeer, Bas (2000), Data Quality and Data Alignement in e-Business, Phd Thesis, BETA Research Institute for Operations Management and Logistics, Eindhoven University of Technology.

[39] Wagner, G.: The Agent-Object-Relationship Meta-Model: Towards a Unified Conceptual View of State and Behaviour. Working Paper. Eindhoven Univ. of Technology, January 2002 (http: //tmitwww.tm.tue.nl/staff/gwagner/AOR.pdf). 